\title{
Clumped isotope thermometry for small sample using isotope dilution method
}

\author{
SANCHITA BANERJEE ${ }^{1}$ AND PROSENJIT GHOSH ${ }^{2}$ \\ ${ }^{1}$ INDIAN INSTITUTE OF SCIENCES \\ ${ }^{2}$ Indian Institute of Science \\ Presenting Author: sanchita933@gmail.com
}

Clumped isotope thermometry for small sample using isotope dilution method

Sanchita Banerjee ${ }^{1}$, Prosenjit Ghosh ${ }^{1,2}$

${ }^{1}$ Centre for Earth Sciences, Indian Institute of Science, Bangalore, India.

${ }^{2}$ Divecha Centre for Climate Change, Indian Institute of Science, Bangalore, India.

Adequate amount of carbonate powder is the prerequisite for determination of temperature during clumped isotope thermometry in natural carbonates. However, some of natural carbonates are available in limited quantity and thereby introduce limitation in analytical protocol. Here we introduce a new isotope dilution method for analysis of smaller amount of carbonate at precision $\pm 0.02 \%$. In this method, we have used two reference carbonate materials (MAR J1- Carrara marble and OMC- Otavi-Meiberg calcite), which are treated as internal laboratory standards and found homogeneous both in $\delta^{13} \mathrm{C}$ and $\delta^{18} \mathrm{O}$ at a precision of 0.002 and $0.003 \%$ [2,3]. These set of reference carbonate powders have $\sim 6.3 \%$ difference in both $\delta^{13} \mathrm{C}$ and $\delta^{18} \mathrm{O}$ and registered significantly different formation temperatures, inferred based on analysis of $\Delta_{47}$ in Absolute reference frame [4]. In this study we mixed variable proportion of these two carbonates having similar grain size and analyse them for clumped and stable isotopic composition using breakseal method [1]. In this experiment, we have treated OMC as unknown whereas, we had prior knowledge of true value for MAR J1. The result suggests a linear trend for $\delta^{13} \mathrm{C}$ and $\delta^{18} \mathrm{O}$ whereas, $\Delta_{47}$ showed non-linearity as demonstrated elsewhere [5]. However, when the mixing is well within $40 \%$ of the target material, the accuracy of predicting the true value of the unknown is well within the acceptable precision of clumped isotope measurement. This provided a most reliable method for analysing carbonate using only $20-40 \%$ of the original sample demand. We have demonstrated validity of this approach using various kind of low temperature carbonates which includes foraminifera, otolith, coral, and ETH-3. This method is similar to isotope dilution approach used for analysis of other isotopes in geochemistry.

Refs: [1] Fosu et al., 2019, RCM 33; [2] Ghosh et al., 2005 RCM 19; [3] Wendeberg et al 2011, RCM 25; [4] Dennis et al 2011, GCA 75; [5] Defliese et al 2015, RCM 29. 